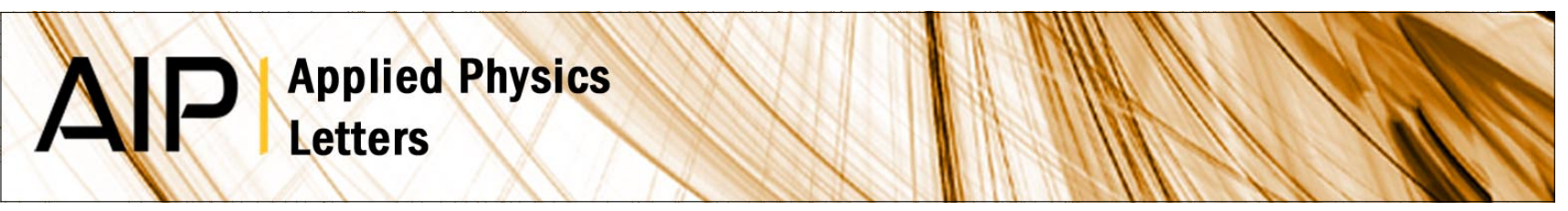

\title{
Large enhancement of the anomalous Hall effect in Co/Pt multilayers sandwiched by $\mathrm{MgO}$ layers
}

S. L. Zhang, J. Teng, J. Y. Zhang, Y. Liu, J. W. Li et al.

Citation: Appl. Phys. Lett. 97, 222504 (2010); doi: 10.1063/1.3522653

View online: http://dx.doi.org/10.1063/1.3522653

View Table of Contents: http://apl.aip.org/resource/1/APPLAB/v97/i22

Published by the AIP Publishing LLC.

Additional information on Appl. Phys. Lett.

Journal Homepage: http://apl.aip.org/

Journal Information: http://apl.aip.org/about/about_the_journal

Top downloads: http://apl.aip.org/features/most_downloaded

Information for Authors: http://apl.aip.org/authors

\section{ADVERTISEMENT}

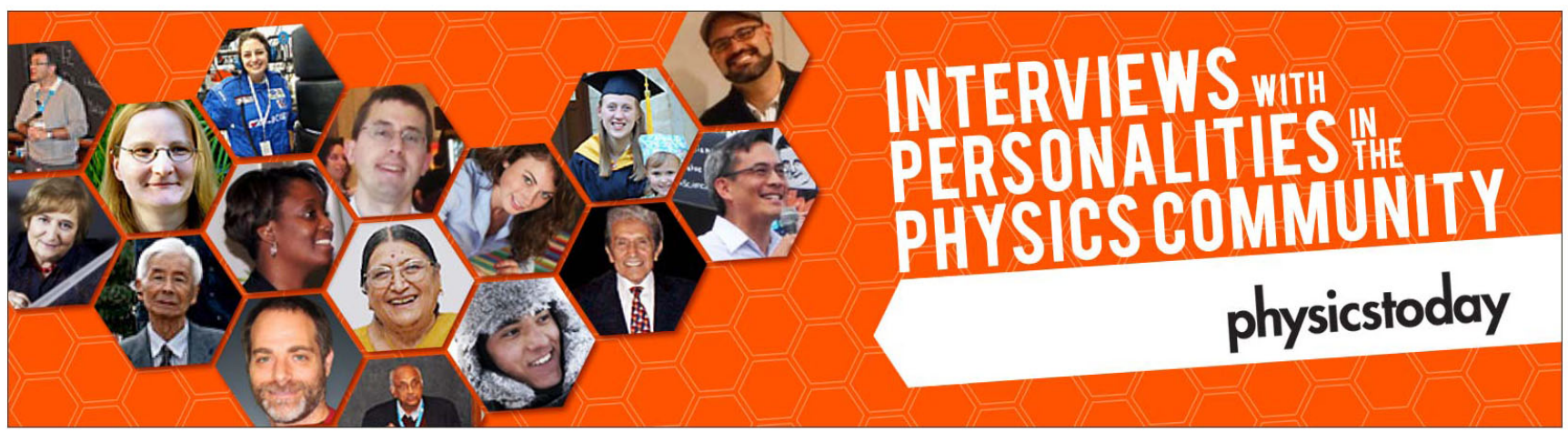




\title{
Large enhancement of the anomalous Hall effect in Co/Pt multilayers sandwiched by MgO layers
}

\author{
S. L. Zhang, ${ }^{1}$ J. Teng, ${ }^{1, a)}$ J. Y. Zhang, ${ }^{1}$ Y. Liu, ${ }^{1}$ J. W. Li, ${ }^{1}$ G. H. Yu, ${ }^{1, a)}$ and S. G. Wang ${ }^{2}$ \\ ${ }^{1}$ Department of Materials Physics and Chemistry, University of Science and Technology Beijing, \\ Beijing 100083, People's Republic of China \\ ${ }^{2}$ State Key Laboratory of Magnetism, Institute of Physics, Chinese Academy of Sciences, Beijing 100190, \\ People's Republic of China
}

(Received 15 September 2010; accepted 5 November 2010; published online 30 November 2010)

\begin{abstract}
We report a large enhanced anomalous Hall effect (AHE) in the Co/Pt multilayers sandwiched by two $\mathrm{MgO}$ layers. The Hall resistivity $\left(\rho_{x y}\right)$ was over an order of magnitude larger than that in pure $\mathrm{Co} / \mathrm{Pt}$ multilayers. By optimizing the thickness of $\mathrm{MgO}$ layers, a high field sensitivity value of 2445 V/A T for Hall sensors was achieved. The enhancement of AHE is mainly attributed to the MgO-Pt interfacial effect. (C) 2010 American Institute of Physics. [doi:10.1063/1.3522653]
\end{abstract}

The anomalous Hall effect (AHE) has been extensively studied during the past few decades because of its controversial physical mechanism ${ }^{1-3}$ and its wide applications. Metallic materials with AHE have been used for magnetic sensors, ${ }^{4-6}$ showing some advantages over semiconductor Hall sensors, such as low resistivity, high operation frequency, and low temperature coefficient. For the AHE sensors, the field sensitivity is one of the primary parameters. A satisfactory sensitivity requires higher AHE resistivity $\left(\rho_{x y}\right)$ and lower hard-axis saturation field $\left(H_{S}\right)$. However, the materials with large $\rho_{x y}$ exhibit high saturation field generally, while the $\rho_{x y}$ in systems with low $H_{S}$ is still of limited magnitudes so far. For example, large $\rho_{x y}$ has been obtained in $\varepsilon-\mathrm{Fe}_{3} \mathrm{~N}$ nanocrystalline films ${ }^{7}$ which is over two orders of magnitude larger than that of bulk $\mathrm{Fe}$, reaching about $20 \mu \Omega \mathrm{cm}$. Unfortunately, its $H_{S}$ is quite large $(>10 \mathrm{kOe})$, suppressing the value of the sensitivity. Ultrathin $\mathrm{CoFe} / \mathrm{Pt}$ multilayers have a low saturation field $(\sim 10 \mathrm{Oe})$, but its $\rho_{x y}$ reaches only $0.6 \mu \Omega \mathrm{cm},{ }^{8}$ just the same level of the thin films of ordinary transition metals $(\sim 1 \mu \Omega \mathrm{cm}) .{ }^{9}$ Although the $\rho_{x y}$ is not very large, the interface anisotropy in $\mathrm{CoFe} / \mathrm{Pt}$ system allows for tuning the $H_{S}$ and the linearity. The sensitivity could reach as high as 1200 V/A T, showing a competition with the best semiconductor Hall sensors. ${ }^{8}$ The multilayered system provides a promising chance for manipulating the anisotropy. Thus, the sensitivity can be substantially improved if $\rho_{x y}$ in this system is greatly enhanced. Recent results have shown that the AHE can be amplified by enhancing the interfacial scattering, which is achieved by structural modifications. This idea was realized in the $[\mathrm{Pt} / \mathrm{Co}]_{5} / \mathrm{Ru} /[\mathrm{Co} / \mathrm{Pt}]_{5}$ multilayers, ${ }^{10}$ where the AHE was much improved due to the strong $\mathrm{Ru} / \mathrm{Co}$ interfacial scattering by introducing $\mathrm{Ru}$ spacer. Therefore, stimulating the interfacial scattering in multilayer systems is an encouraging approach for developing high-sensitivity AHE sensors.

Comparing with the metal-metal interface, the amorphous insulator-metal interface can increase the AHE more significantly because the amorphous insulator-metal interface contains more defects for additional scattering of electrons. ${ }^{11}$ Recently, interfaces consisting of the insulator $\mathrm{MgO}$ are

\footnotetext{
a) Author to whom correspondence should be addressed. Electronic mail: ghyu@mater.ustb.edu.cn.
}

shown to have a strong scattering capability for the tunnel magnetoresistance ${ }^{12}$ and anisotropic magnetoresistance ${ }^{13}$ materials. In this paper, we report a method to enhance the AHE by introducing $\mathrm{MgO}$ (amorphous)-metal interfaces in the $\mathrm{Co} / \mathrm{Pt}$ multilayers with tunable anisotropy for highsensitivity Hall sensors.

Multilayered samples with structure of $\mathrm{Pt}(0.6) /$ $[\mathrm{Co}(0.4) / \mathrm{Pt}(1.2)]_{n}$ (in nanometer) (pure $\mathrm{Co} / \mathrm{Pt}$ multilayers, called $\mathrm{S} 1), \quad \mathrm{MgO}\left(t_{\mathrm{MgO}}^{\mathrm{sub}}\right) / \mathrm{Pt}(0.6) /[\mathrm{Co}(0.4) / \mathrm{Pt}(1.2)]_{m} /$ $\mathrm{MgO}\left(t_{\mathrm{MgO}}^{\text {top }}\right)$ (in nanometer) $(\mathrm{Co} / \mathrm{Pt}$ multilayers sandwiched
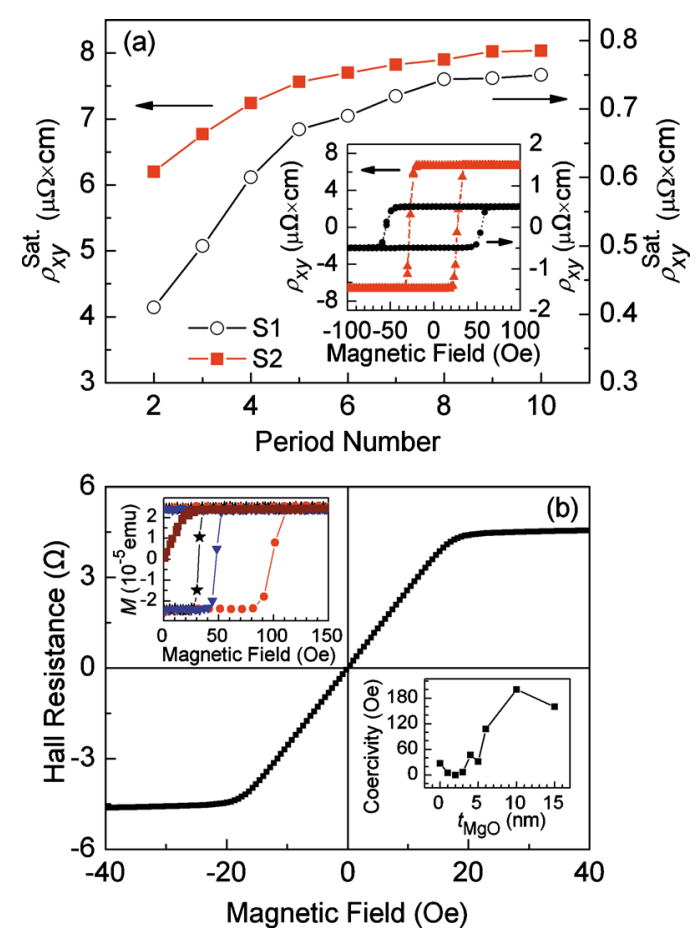

FIG. 1. (Color online) (a) Saturation Hall resistivity $\left(\rho_{x y}^{\text {sat }}\right)$ as a function of period number $n$ for S1 (empty circle) and $\rho_{x y}^{\text {sat }}$ as a function of $m$ for S2 $\left(t_{\mathrm{MgO}}^{\mathrm{sub}}=t_{\mathrm{MgO}}^{\text {top }}=5 \mathrm{~nm}\right)$ (solid rectangle). Inset: Hall resistivity as a function of magnetic field measured at RT for multilayers $n=3$ (circle) and $m=3$, $\left(t_{\mathrm{MgO}}^{\mathrm{sub}}=t_{\mathrm{MgO}}^{\mathrm{top}}=5 \mathrm{~nm}\right)$ (triangle). (b) Hall resistance as a function of the field for the multilayer $\mathrm{MgO}(3) / \mathrm{Pt}(0.6) /[\mathrm{Co}(0.4) / \mathrm{Pt}(1.2)]_{3} / \mathrm{MgO}(1)$ (in nanometer). Right bottom inset: the coercivity as a function of $t_{\mathrm{MgO}}\left(t_{\mathrm{MgO}}^{\mathrm{sub}}\right.$ $\left.=t_{\mathrm{MgO}}^{\text {top }}\right)$ for S2, $m=3$. Left top inset: hysteresis loops for S2, $m=3$ with $t_{\mathrm{MgO}}^{\text {sub }}=3 \mathrm{~nm}$ and varying $t_{\mathrm{MgO}}^{\text {top }}\left(\right.$ rectangle: $t_{\mathrm{MgO}}^{\text {top }}=1.5 \mathrm{~nm}$, star: $t_{\mathrm{MgO}}^{\text {top }}=2 \mathrm{~nm}$, triangle: $t_{\mathrm{MgO}}^{\text {top }}=3 \mathrm{~nm}$, and circle: $t_{\mathrm{MgO}}^{\text {top }}=4 \mathrm{~nm}$ ). 


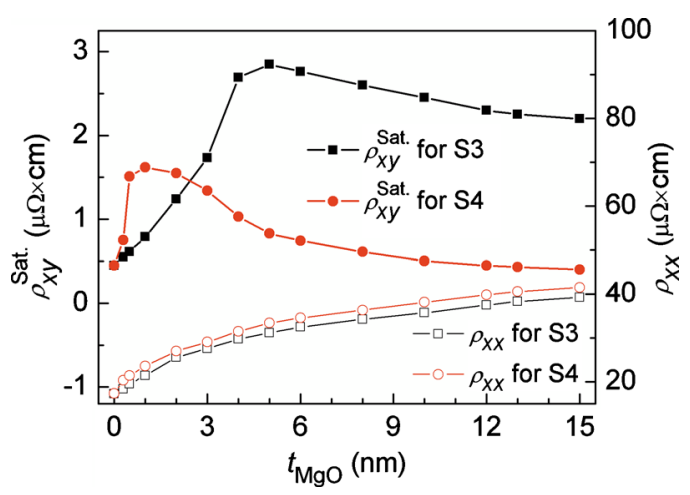

FIG. 2. (Color online) Saturation Hall resistivity $\rho_{x y}^{\text {sat }}$ and longitudinal resistivity $\rho_{x x}$ as a function of the $\mathrm{MgO}$ thickness for samples S3 and S4.

by $\mathrm{MgO}$ layers, called $\mathrm{S} 2), \quad \mathrm{MgO}\left(\mathrm{MgO}_{\mathrm{MgO}}^{\mathrm{sub}}\right) / \mathrm{Pt}(0.6) /$ $[\mathrm{Co}(0.4) / \mathrm{Pt}(1.2)]_{3}$ (in nanometer) $(\mathrm{Co} / \mathrm{Pt}$ multilayers with $\mathrm{MgO}$ underneath, called $\mathrm{S} 3)$, and $\mathrm{Pt}(0.6) /[\mathrm{Co}(0.4) /$ $\mathrm{Pt}(1.2)]_{3} / \mathrm{MgO}\left(t_{\mathrm{MgO}}^{\text {top }}\right)$ (in nanometer) $(\mathrm{Co} / \mathrm{Pt}$ multilayers with $\mathrm{MgO}$ on the top, called S4) were deposited onto thermally oxidized $\mathrm{Si}$ wafers at room temperature (RT) by magnetron sputtering. The thickness of the amorphous $\mathrm{MgO}$ layer $\left(t_{\mathrm{MgO}}^{\mathrm{sub}}\right.$ and $t_{\mathrm{MgO}}^{\text {top }}$ ) varies from 1 to $15 \mathrm{~nm}$, and the period number ( $m$ and $n$ ) varies from 2 to 10 , respectively. The base pressure was prior to $1.0 \times 10^{-5} \mathrm{~Pa}$, and the Ar pressure was kept at $0.2 \mathrm{~Pa}$ during sputtering. The deposition rate of $\mathrm{Co}, \mathrm{Pt}$, and $\mathrm{MgO}$ is $0.071,0.068$, and $0.005 \mathrm{~nm} / \mathrm{s}$, respectively. Standard optical photolithography was applied to fabricate the Hall bars for the transport measurement with physical property measurement system (produced by Quantum Design in USA) using the standard four-probe technique. Magnetic properties were also measured by alternating gradient magnetometer.

The inset of Fig. 1(a) shows the AHE resistivity as a function of the magnetic field measured at RT for multilayers of $\mathrm{Pt}(0.6) /[\mathrm{Co}(0.4) / \mathrm{Pt}(1.2)]_{3}$ (in nanometer) (circle) and $\mathrm{MgO}(5) / \mathrm{Pt}(0.6) /[\mathrm{Co}(0.4) / \mathrm{Pt}(1.2)]_{3} / \mathrm{MgO}(5)$ (in nanometer) (triangle). It shows that the Hall resistivity increases significantly when the pure $\mathrm{Co} / \mathrm{Pt}$ multilayers are sandwiched by $\mathrm{MgO}$ layers. The saturation Hall resistivity $\left(\rho_{x y}^{\text {sat }}\right)$ value reaches $6.7 \mu \Omega \mathrm{cm}$, whereas it is only $0.5 \mu \Omega \mathrm{cm}$ in the sample without $\mathrm{MgO}$ layers. The enhancement is approximately 13 times by introducing $\mathrm{MgO}$ layers on both sides. Furthermore, the period number of the Co/Pt bilayers $(n$ $=m=2-10)$ slightly affects the magnitude of $\rho_{x y}^{\text {sat }}$ both in S1 and S2, shown in Fig. 1(a), indicating that the enhancement of $\rho_{x y}$ is due to the interfacial effect at the $\mathrm{MgO}-\mathrm{Pt}$ interfaces instead of that at the Co-Pt interfaces in the multilayers. The right bottom inset of Fig. 1(b) shows the coercivity of the multilayers $\mathrm{MgO}\left(t_{\mathrm{MgO}}\right) / \mathrm{Pt}(0.6) /[\mathrm{Co}(0.4) / \mathrm{Pt}(1.2)]_{3} /$ $\mathrm{MgO}\left(t_{\mathrm{MgO}}\right)$ (in nanometer) as a function of $t_{\mathrm{MgO}}$. It shows that the coercivity changes nonlinearly with the $\mathrm{MgO}$ thickness, suggesting a sensitive anisotropy in the multilayers. The left top inset shows the perpendicular hysteresis loops for the multilayers $\mathrm{MgO}\left(t_{\mathrm{MgO}}^{\mathrm{sub}}\right) / \mathrm{Pt}(0.6) /[\mathrm{Co}(0.4) /$ $\mathrm{Pt}(1.2)]_{3} / \mathrm{MgO}\left(t_{\mathrm{MgO}}^{\text {top }}\right)$ (in nanometer) with $t_{\mathrm{MgO}}^{\text {sub }}=3 \mathrm{~nm}$ and various $t_{\mathrm{MgO}}^{\text {top }}$ For samples with $t_{\mathrm{MgO}}^{\mathrm{sub}}$ being around $3 \mathrm{~nm}$ and $t_{\mathrm{MgO}}^{\text {top }}$ being around $1.5 \mathrm{~nm}$, the magnetization loop shows an anisotropy shift from out-of-plane to in-plane. Thus, a high value of sensitivity with large $\rho_{x y}$ and low hard-axis $H_{S}$ can be obtained by optimizing the thicknesses of $\mathrm{MgO}$ layers. Figure 1(b) shows the Hall resistance loop at RT for the
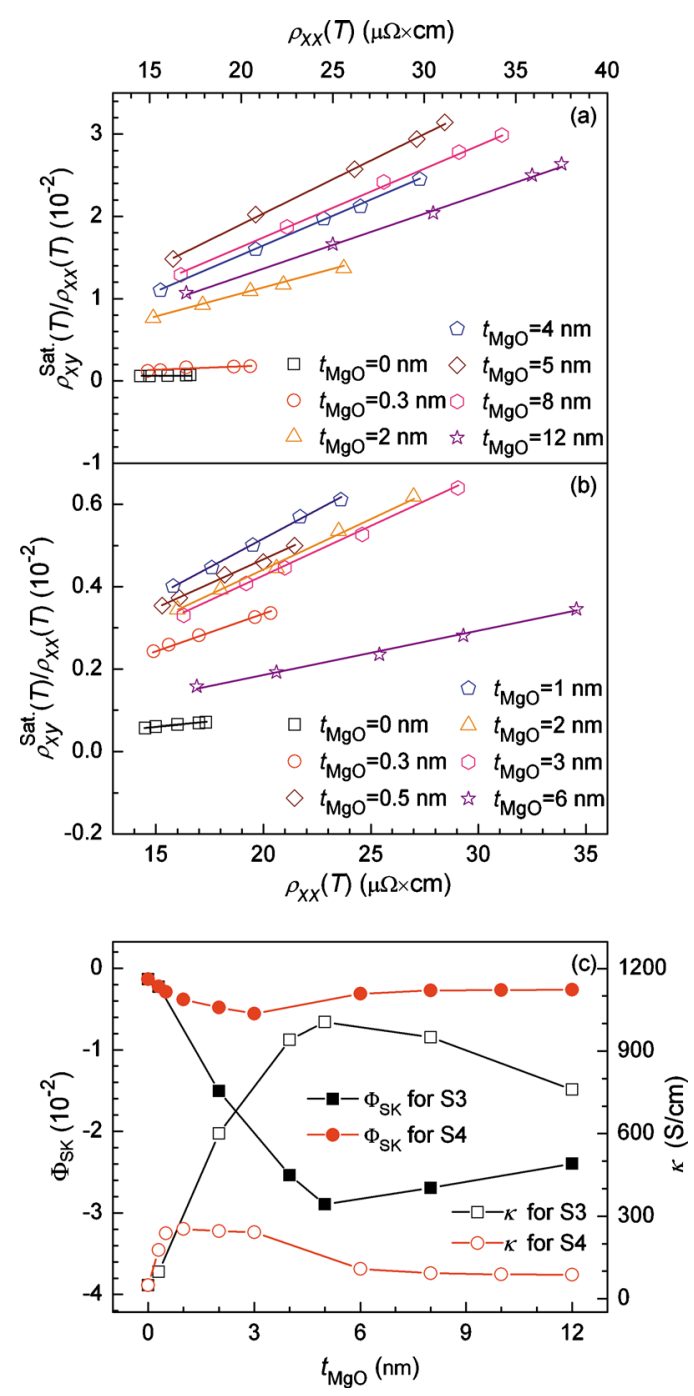

FIG. 3. (Color online) (a) $\rho_{x y}^{\text {sat }}(T) / \rho_{x x}(T)$ vs $\rho_{x x}(T)$ for $\mathrm{S} 3$ where each sample's $\rho_{x y}^{\text {sat }}(T)$ and $\rho_{x x}(T)$ were plotted in several different temperatures. (b) $\rho_{x y}^{\text {sat }}(T) / \rho_{x x}(T)$ vs $\rho_{x x}(T)$ for S4. (c) $\Phi_{\text {sk }}$ and $\kappa$ as a function of the $\mathrm{MgO}$ thickness for S3 and S4.

sample $\quad \mathrm{MgO}(3) / \mathrm{Pt}(0.6) /[\mathrm{Co}(0.4) / \mathrm{Pt}(1.2)]_{3} / \mathrm{MgO}(1) \quad$ (in nanometer). The Hall resistance is increased to $4.5 \mathrm{~V} / \mathrm{A}$, and the saturation field is only approximately 20 Oe. The Hall resistance loop shows a good linearity with field sensitivity as high as $2445 \mathrm{~V} / \mathrm{A} \mathrm{T}$, which is about two times larger than that of the current best metallic Hall sensors (1200 V/A T). ${ }^{8}$

As the top and the bottom $\mathrm{MgO}$ layers have different influences on the interfacial crystalline morphology, it is necessary to investigate them, respectively. The focus is given on the two separated series of samples: S3 and S4. Figure 2 shows $\rho_{x y}^{\text {sat }}$ and the longitudinal resistivity $\left(\rho_{x x}\right)$ as a function of the thickness of $\mathrm{MgO}$ layer in $\mathrm{S} 3\left(t_{\mathrm{MgO}}^{\text {sub }}\right)$ and $\mathrm{S} 4\left(t_{\mathrm{MgO}}^{\text {top }}\right)$, respectively. The $\rho_{x x}$ for both $\mathrm{S} 3$ and $\mathrm{S} 4$ shows a monotonic increase slightly with increasing the $\mathrm{MgO}$ thickness. The $\rho_{x y}^{\text {sat }}$ increases significantly with increasing $t_{\mathrm{MgO}}$ for $\mathrm{S} 3$ and $\mathrm{S} 4$, with the maximum value at $t_{\mathrm{MgO}}^{\mathrm{sub}}=5 \mathrm{~nm}$ and $t_{\mathrm{MgO}}^{\mathrm{top}}=2 \mathrm{~nm}$ for S3 and S4, respectively. Then $\rho_{x y}^{\text {sat }}$ decreases with further increasing $t_{\mathrm{MgO}}$. For the optimized values of $t_{\mathrm{MgO}}$ in each series, $\rho_{x v}^{\text {sat }}$ increases to 2.85 and $1.62 \mu \Omega \mathrm{cm}$ compared with $0.5 \mu \Omega \mathrm{cm}$ in multilayers without $\mathrm{MgO}$ layers. It shows six and three times larger in magnitude than that in the reference sample $\left(t_{\mathrm{MgO}}=0 \mathrm{~nm}\right)$. It can be known by comparing $\mathrm{S} 3$ and 
$\mathrm{S} 4$ that the top and the bottom $\mathrm{MgO}$ layers make different contributions to AHE. The highest value of $\rho_{x y}$ is obtained in S2, indicating that the enhancement of the AHE is attributed to the interfacial effect at both the $\mathrm{MgO} / \mathrm{Pt}$ and the $\mathrm{Pt} / \mathrm{MgO}$ interfaces.

In general, the AHE has both intrinsic and extrinsic origins, and $\rho_{x y}^{\text {sat }}$ can be expressed as

$$
\rho_{x y}^{\mathrm{sat}}=\Phi_{\mathrm{sk}} \rho_{x x}+\kappa \rho_{x x}^{2},
$$

where the linear term with parameter $\Phi_{\mathrm{sk}}$ stands for the extrinsic skew scattering ${ }^{14}$ and the parameter of the quadratic term $\kappa$ incorporates two parts: one is the intrinsic conductivity $\kappa^{\text {int }}$ which comes from the band structure effect, ${ }^{15}$ the other is the side-jump conductivity $\kappa^{\mathrm{sj}}$ which accumulates all the other extrinsic contributions to $\rho_{x y}^{\text {sat }},{ }^{16}$ and can be boosted by interface scattering usually in a multilayer system, ${ }^{17}$ i.e., $\kappa=\kappa^{\mathrm{int}}+\kappa^{\mathrm{sj}}$. Recently it is known from the work of Seemann et $a .^{3}$ that the proportion of $\kappa^{\mathrm{int}}$ and $\kappa^{\mathrm{sj}}$ to $\kappa$ is determined mainly by the spin-orbit coupling strength in the material. Often, $\Phi_{\mathrm{sk}}$ and $\kappa$ can be experimentally distinguished in a plot of $\rho_{x y}^{\text {sat }} / \rho_{x x}$ versus $\rho_{x x}$ by varying $\rho_{x y}^{\text {sat }}$ and $\rho_{x x}$ through temperature changes.

Figures 3(a) and 3(b) show $\rho_{x y}^{\text {sat }}(T) / \rho_{x x}(T)$ versus $\rho_{x x}(T)$ for S3 and S4. Figure 3(c) shows the values of $\Phi_{\text {sk }}$ and $\kappa$ derived from Figs. 3(a) and 3(b) as a function of $t_{\mathrm{MgO}}$ for samples S3 and S4. The maximum value of $\kappa$ in S3 and S4 is about 21 and five times larger than that in pure $\mathrm{Co} / \mathrm{Pt}$ multilayers, respectively. The larger $\kappa$ is, the higher the AHE is. For $\Phi_{\mathrm{sk}}$, the maximum absolute value of $\Phi_{\mathrm{sk}}$ is 25 and four times larger than those in pure $\mathrm{Co} / \mathrm{Pt}$ multilayer for $\mathrm{S} 3$ and S4, respectively. However, the skew scattering contribution is always negative in our samples. Thus, the large enhanced AHE is dominated by the enhanced $\kappa$, which is determined by both $\kappa^{\mathrm{sj}}$ and $\kappa^{\mathrm{int}}$. It is known that the scattering event contributes to both $\Phi_{\mathrm{sk}}$ and $\kappa$, while the intrinsic origin only makes contribution to $\kappa$. Since the absolute values of $\Phi_{\mathrm{sk}}$ and $\kappa$ increase/decrease synchronously as $t_{\mathrm{MgO}}$ increases, indicating that the enhanced $\kappa$ is not only due to the intrinsic mechanism, but also results from the scattering effect (side jump mechanism) at the MgO-Pt interface. This enhancement of $\kappa$ can be mainly attributed to the $\mathrm{MgO}-\mathrm{Pt}$ interfacial effect.
In conclusion, we have presented a detailed study of the AHE for $\mathrm{Co} / \mathrm{Pt}$ multilayers sandwiched by two insulator amorphous $\mathrm{MgO}$ layers. The AHE for this multilayer is much larger than that of the system without $\mathrm{MgO}$ layers. By optimizing the thicknesses of $\mathrm{MgO}$ layers, an approach that enables generation of high-sensitivity AHE sensors is provided. The enhanced AHE in our multilayers is attributed to the large $\kappa$ contribution, which is mainly due to the $\mathrm{MgO}-\mathrm{Pt}$ interfacial effect.

This work is supported by the National High Technology Research and Development Program 863 (Grant No. 2007AA032310), the National Basic Research Program of China (Grant No. 2009CB929203), and the National Natural Science Foundation of China (Grant Nos. 51071023, 50871014, 50831002, 50901007, and 50972163).

${ }^{1}$ Y. Tian, L. Ye, and X. Jin, Phys. Rev. Lett. 103, 087206 (2009).

${ }^{2}$ E. Roman, Y. Mokrousov, and I. Souza, Phys. Rev. Lett. 103, 097203 (2009)

${ }^{3}$ K. M. Seemann, Y. Mokrousov, A. Aziz, J. Miguel, F. Kronast, W. Kuch, M. G. Blamire, A. T. Hindmarch, B. J. Hickey, I. Souza, and C. H. Marrows, Phys. Rev. Lett. 104, 076402 (2010).

${ }^{4}$ T. R. McGuire, R. J. Gambino, and R. C. Taylor, J. Appl. Phys. 48, 2965 (1977).

${ }^{5}$ A. B. Pakhomov, X. Yan, and B. Zhao, Appl. Phys. Lett. 67, 3497 (1995).

${ }^{6}$ G. X. Miao and G. Xiao, Appl. Phys. Lett. 85, 73 (2004).

${ }^{7}$ Y. H. Cheng, R. K. Zheng, H. Liu, Y. Tian, and Z. Q. Li, Phys. Rev. B 80, 174412 (2009).

${ }^{8}$ Y. Zhu and J. W. Cai, Appl. Phys. Lett. 90, 012104 (2007).

${ }^{9}$ A. Gerber, A. Milner, M. Karpovsky, B. Lemke, H. U. Habermeier, J. Tuaillon-Combes, M. Negrier, O. Boisron, P. Melinon, and A. Perez, J. Magn. Magn. Mater. 242-245, 90 (2002).

${ }^{10}$ J. Zhao, Y. J. Wang, X. F. Han, S. Zhang, and X. H. Ma, Phys. Rev. B 81, 172404 (2010).

${ }^{11}$ N. Ryzhanova, A. Vedyayev, A. Pertsova, and B. Dieny, Phys. Rev. B 80 024410 (2009).

${ }^{12}$ A. Manchon, C. Ducruet, L. Lombard, S. Auffret, B. Rodmacq, B. Dieny, S. Pizzini, J. Vogel, V. Uhlir, M. Hochstrasser, and G. Panaccione, J. Appl. Phys. 104, 043914 (2008).

${ }^{13}$ L. Ding, J. Teng, X. C. Wang, C. Feng, Y. Jiang, G. H. Yu, S. G. Wang, and R. C. C. Ward, Appl. Phys. Lett. 96, 052515 (2010).

${ }^{14}$ J. Smit, Physica 24, 39 (1958).

${ }^{15}$ R. Karplus and J. M. Luttinger, Phys. Rev. 95, 1154 (1954).

${ }^{16}$ L. Berger, Phys. Rev. B 2, 4559 (1970).

${ }^{17}$ C. L. Canedy, X. W. Li, and G. Xiao, Phys. Rev. B 62, 508 (2000). 